\title{
Preoperative Sterispon embolisation to facilitate wide excision of mesenchymal chondrosarcoma of the ribs
}

\author{
RJH EMERY, PLC SMITH, T TREASURE \\ From the Middlesex Hospital, London
}

Mesenchymal chondrosarcoma is a rare tumour occurring most frequently either in the ribs or in the jaw. It is generally accepted that wide excision is the treatment of choice. In the chest, however, the tumour can be very large and vascular, being fed by several intercostal arteries. We have used absorbable gelatin sponge (Sterispon, Allen and Hanburys) to produce embolisation of these arteries on the day of operation with the object of reducing vascularity before wide surgical excision.

\section{Case report}

A 21 year old caucasian man presented to another surgical firm with a $5 \mathrm{~cm}$ diameter opacity in the left chest that had been found on a routine chest radiograph, and underwent exploratory left thoractomy. A vascular tumour was found at the postero-medial end of the left 7 th rib and was thought to be a ganglioneuroma; because of the tumour site and vascularity and the knowledge that ganglioneuromas do not grow after puberty, it was left in situ.

Three years later, at the age of 24 , he presented again with recurrent sharp pain in the left chest. Examination showed nothing abnormal but chest radiographs showed a large mass in the left chest, now $11 \mathrm{~cm}$ in diameter. His alkaline phosphatase activity was raised at $293 \mathrm{IU} / \mathrm{l}$, the erythrocyte sedimentation rate was $2 \mathrm{~mm}$ in one hour (West), and the results of all other routine blood investigations were within the normal range. An arch aortogram with selective catheterisation of the three large left intercostal arteries affected showed the tumour to be extremely vascular (fig 1). Computed tomography of the left chest showed a large left paravertebral mass, which was partially calcified. There was no evidence that the vertebral bodies or neural foramina were affected.

On the morning of his second thoracotomy the patient underwent embolisation of the relevant intercostal arteries feeding the vascular tumour (fig 2). This was carried out with shredded Sterispon using a Seldinger technique via the right femoral artery. Immediately after embolisation an extensive left posterolateral thoracotomy was carried out. After adherent lung had been dissected away from the tumour it became evident that the tumour had invaded the 5 th, 6 th, 7 th, and 8 th ribs. Wide excision of the tumour was carried out, removing a plate of the affected ribs. The very

Address for reprint requests: Mr T Treasure, Middlesex Hospital, London W1N 8AA.

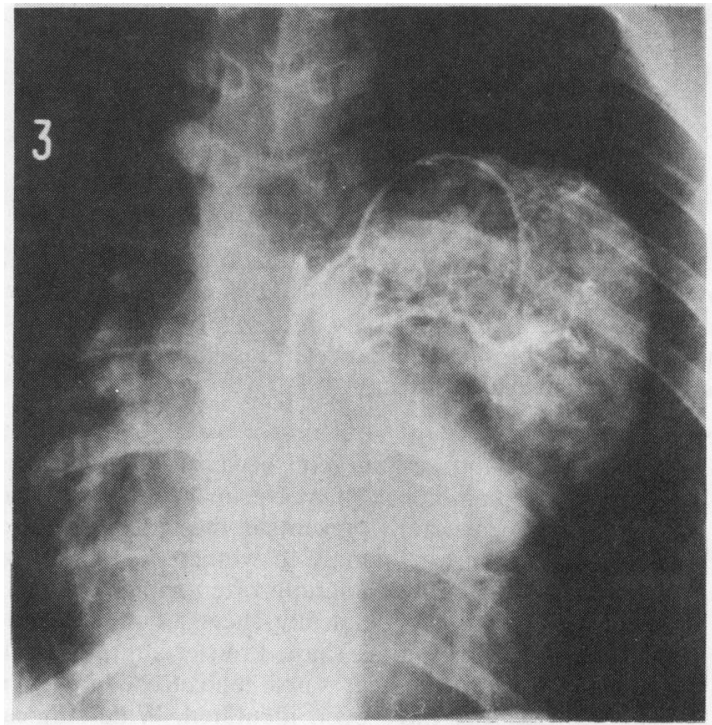

Fig 1 Angiogram of one of the three left intercostal arteries supplying the tumour.

large intercostal arteries were divided between clips and vascularity was not a problem. The chest wall deficit was largely covered by the scapula and chest wall reconstruction was not required. After routine closure the patient was ventilated electively overnight in the intensive care unit. The further postoperative course was uneventful and he was discharged well after 14 days.

Histological examination showed the tumour to be a mesenchymal chondrosarcoma characterised by highly cellular zones composed of anaplastic small cells and islands of relatively benign appearing chondroid substance, which were calcified and even ossified in some areas.

At follow up four months after operation the patient was well and playing active competitive sport, with no signs of recurrence; this was confirmed by computed tomography.

\section{Discussion}

Mesenchymal chondrosarcoma was first described in 1959 by Liechenstein and Bernstein. ${ }^{1}$ Further cases have since been described, notably by Dahlin and Henderson, ${ }^{2}$ Dowling, ${ }^{3}$ and Salvador et al..$^{4}$ It is of particular interest and 


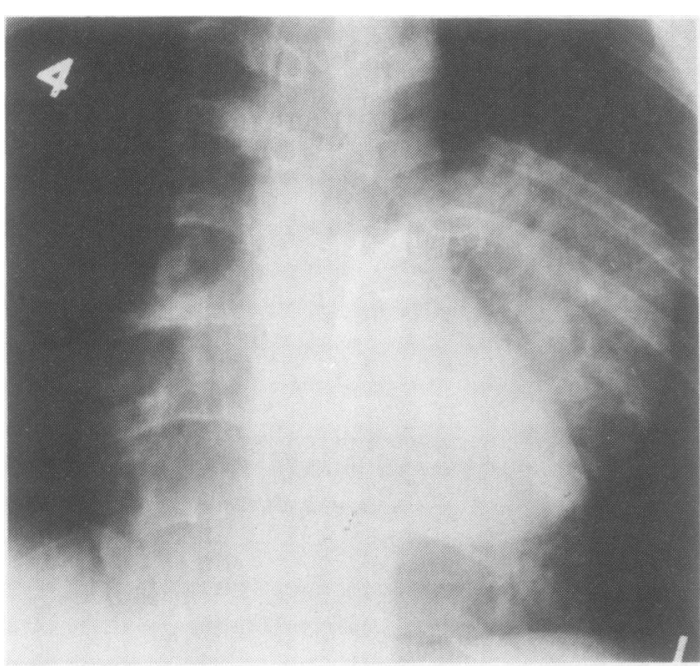

Fig 2 The same intercostal artery after Sterispon embolisation; the reduction of vascularity and the sharp cut off point in the major artery can be seen. Similar pictures were obtained after the other two large feeding intercostal arteries had been embolised.

relevance to the thoracic surgeon because, although it is rare, one of the commoner sites of primary tumour is the ribs. The tumour generally presents in the second or third decade of life, more commonly in women, with pain or swelling or both. Chest radiograph often shows irregular stippled calcification. Histologically, sheets of undifferentiated small cells with spindle shaped nuclei alternate with chondroid islets. ${ }^{5}$ The clinical course tends to be long even if distant metastases have been identified. Wide surgical excision is the treatment of choice, holding out hope of the longest survival. ${ }^{4}$ Radiotherapy probably has little place in the primary treatment or in palliation.

The revelant intercostal feeding arteries were embolised for us by Dr Maurice Raphael on the day of operation. The subsequent surgery was not complicated by evident vascularity and, while we have no control procedure which is directly comparable, we believe that embolisation made wide excision a less formidable surgical undertaking. ${ }^{6}$ The technique of embolising the intercostal arteries with shredded Sterispon was not difficult in the radiology department with the help of imaging techniques, though pain in the tumour immediately after embolisation may be a problem and attention to adequate sedation and analgesia between embolisation and thoractomy is important.?

Though mesenchymal chondrosarcoma of the chest wall may appear to offer a great challenge to surgical removal because of its size and vascularity, we believe that this always should at least be attempted, as it is often a slow growing and partially encapsulated tumour. Preoperative embolisation can render the vascularity manageable, and in addition the accompanying pain in the chest wall can be relieved. Even in the presence of a solitary secondary deposit survival may be of many years' duration. ${ }^{4}$

We wish to thank Dr Maurice Raphael, department of radiology, for his permission to reproduce the radiographs.

\section{References}

' Leichenstein L, Bernstein D. Unusual benign and malignant chondroid tumours of bone: a survey of some mesenchymal cartilage tumours and malignant chondroblastic tumours, including a few multicentric ones, as well as many atypical benign chondroblastomas and chondromyxoid fibromas. Cancer 1959;12:1142-57.

${ }^{2}$ Dahlin DC, Henderson ED. Mesenchymal chondrosarcoma: further observations of a new entity. Cancer 1962;15:410-7.

${ }^{3}$ Dowling EA. Mesenchymal chondrosarcoma. J Bone Joint Surg 1964;46A:747-54.

${ }^{4}$ Salvador AH, Beabout JW, Dahlin DC. Mesenchymal chondrosarcoma: observations on 30 new cases. Cancer 1971;28:605-15.

${ }^{5}$ Hutter RVP, Foot FW jun, Francis KC, Sherman R. Primitive multipotential primary sarcoma of bone. Cancer 1966;19:125.

- Feldman F, Casarella WJ, Dick HM, Hollander BA. Selective intra-arterial embolisation of bone tumours. Radiology 1975;123:130-9.

' Chuang VP, Wallace S. Current status of transcatheter management of neoplasms. Journal of Cardiovascular Interventional Radiology 1980;3:256-65. 